Supporting information

\title{
Multiphoton Excitation of Upconverting Nanoparticles in Pulsed Regime.
}

\author{
Jose Hodak ${ }^{1}$, Zhijun Chen ${ }^{2}$, Si $W u^{2}$ and Roberto Etchenique ${ }^{1 *}$
}

1. Departamento de Química Inorgánica, Analítica y Química Física, INQUIMAE, Facultad de Ciencias Exactas y Naturales, Universidad de Buenos Aires, Ciudad Universitaria Pabellón 2 AR1428EHA Buenos Aires, Argentina. 2. Max Planck Institute for Polymer Research, Ackermannweg 10, 55128 Mainz, Germany.

Email: rober@qi.fcen.uba.ar

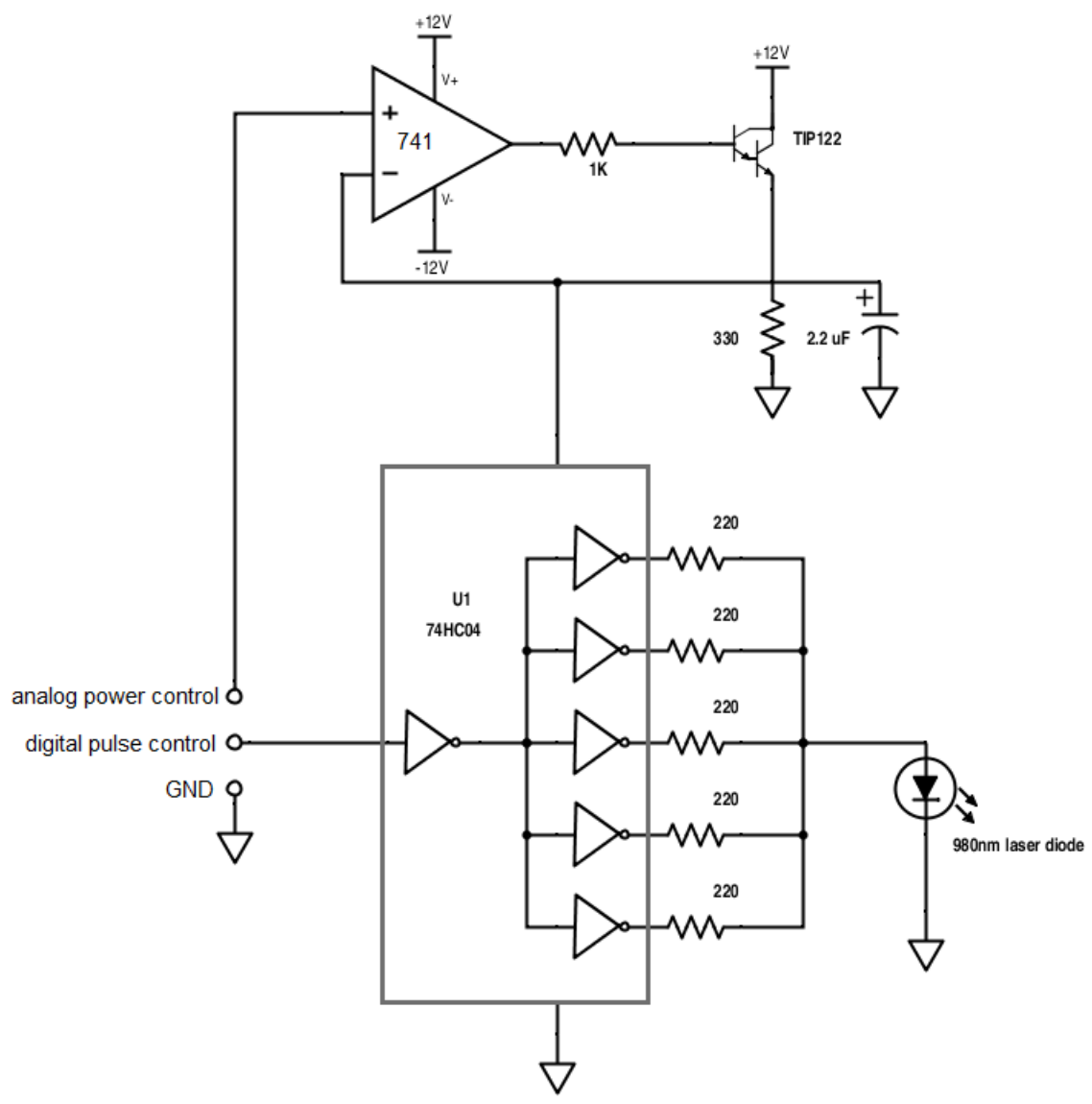

Figure S1. Diagram of the electronic circuit used to control both power and pulse duration of the excitation laser diode. 

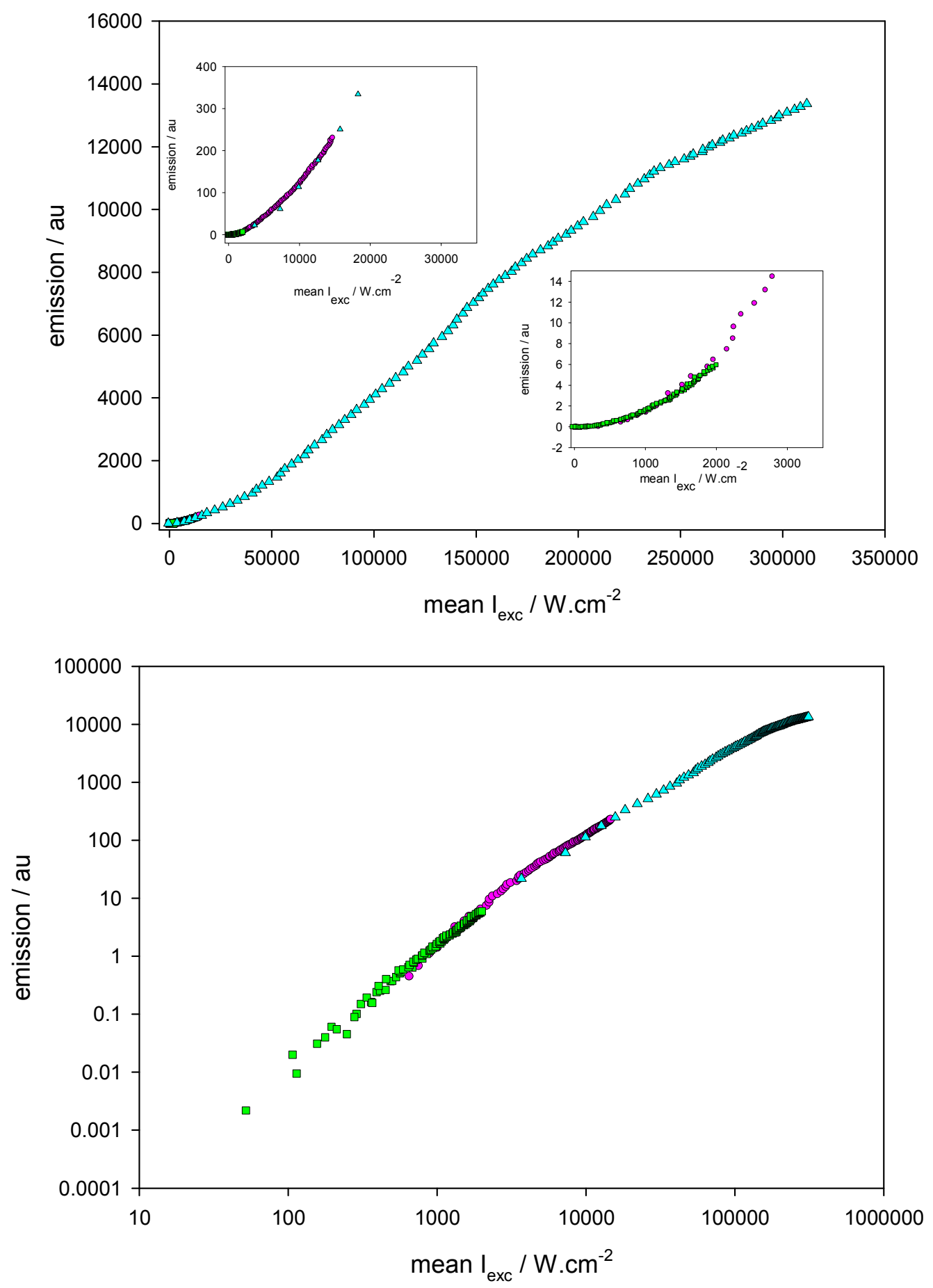

Figure S2. Dependence of the ${ }^{4} \mathrm{~F}_{9 / 2}{ }^{4} \mathrm{I}_{15 / 2}$ emission on the excitation, covering several orders of magnitude of average power density. Top: Linear plot. Bottom: Log-Log plot. Green squares: instantaneous power $=2 \mathrm{~kW} / \mathrm{cm}^{2}$. Pink circles: $15 \mathrm{~kW} / \mathrm{cm}^{2}$. Cyan triangles: $310 \mathrm{~kW} / \mathrm{cm}^{2}$. At each power the duty cycle is varied from 0 to $100 \%$ at $\mathrm{f}=100$ $\mathrm{Hz}$. 

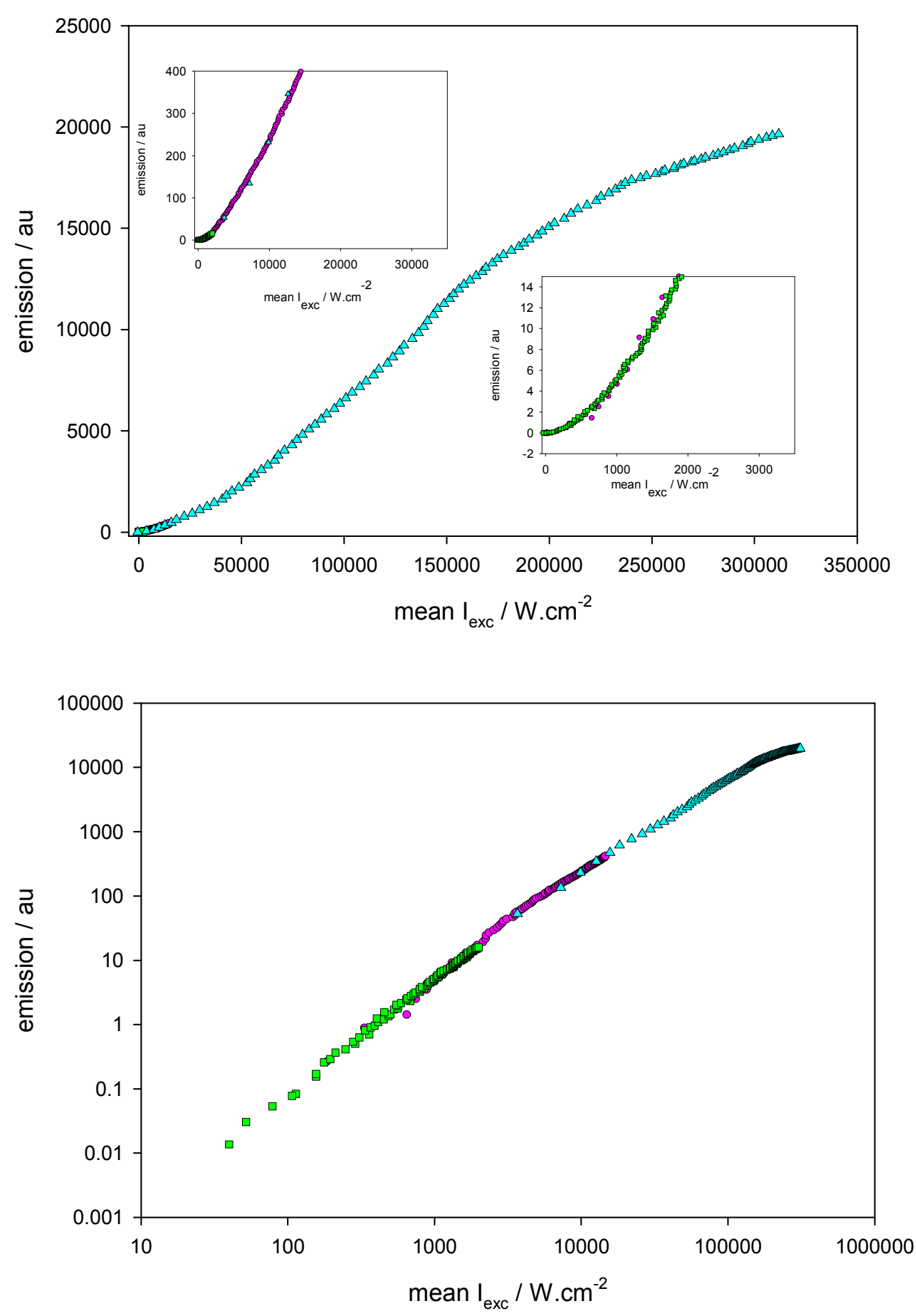

Figure S3. Dependence of the ${ }^{4} \mathrm{~S}_{3 / 2}{ }^{4} \mathrm{I}_{15 / 2}$ band emission on the excitation, covering several orders of magnitude of average power density. Top: Linear plot. Bottom: LogLog plot. Green squares: instantaneous power $=2 \mathrm{~kW} / \mathrm{cm}^{2}$. Pink circles: $15 \mathrm{~kW} / \mathrm{cm}^{2}$. Cyan triangles: $310 \mathrm{~kW} / \mathrm{cm}^{2}$. At each power the duty cycle is varied from 0 to $100 \%$ at $\mathrm{f}=100 \mathrm{~Hz}$. 


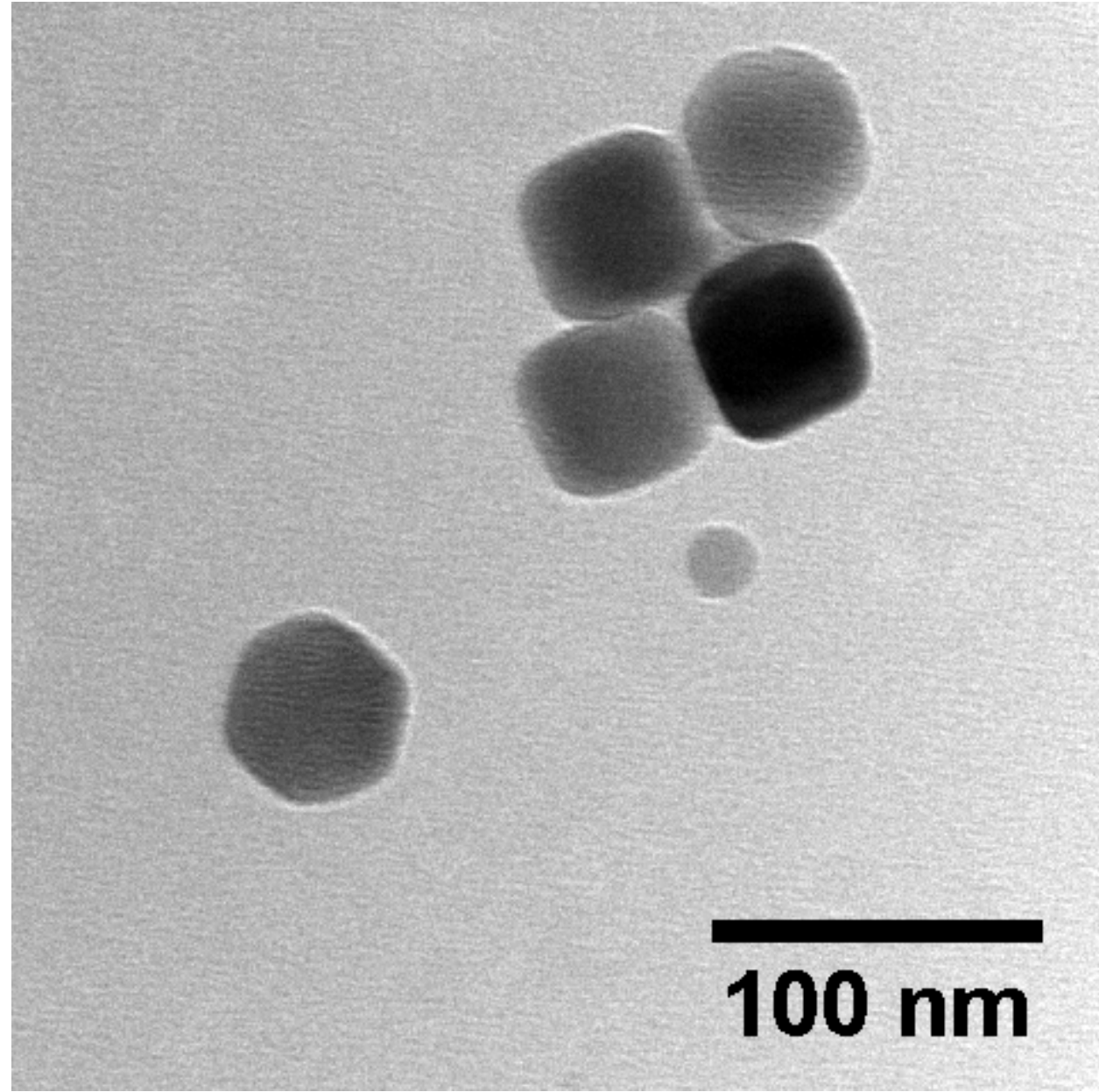

Figure S4. TEM microphotography of the Er-containing UCNPs. . 
For a simple $2 \mathrm{P}$ absorption process we have two states $\mathrm{S}_{0}$ and $\mathrm{S}_{1}$. The particle is excited to $\mathrm{S}_{1}$, from which it can decay by a non-radiative pathway with rate $k_{N R}$ and by an emissive pathway with rate $k_{R}$.

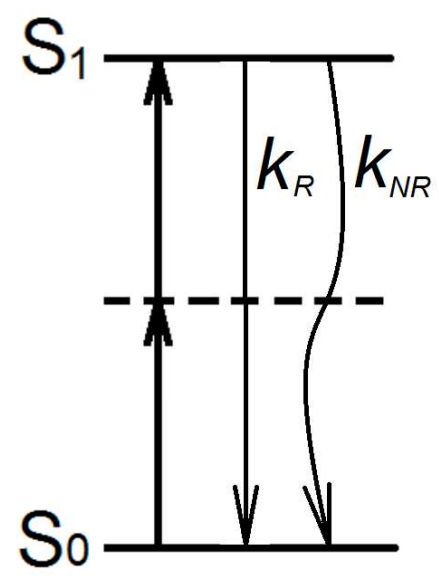

Figure S5: Scheme of the 2P fluorescence process.

The set of differential equations that describe this system can be written as:

$$
\begin{aligned}
\frac{d N_{0}}{d t} & =-\frac{1}{2} I_{0}\left(1-\frac{1}{1+\sigma_{2 P} I_{0} N_{0}}\right)+k_{R} N_{1}+k_{N R} N_{1} \\
\frac{d N_{1}}{d t} & =-\frac{d N_{0}}{d t}
\end{aligned}
$$


Where $I_{0}$ is the excitation power density in photon. $\mathrm{cm}^{-2} \cdot \mathrm{s}^{-1}$ and $\sigma_{2 \mathrm{P}}$ is the two-photon absorption cross section in units of $\mathrm{cm}^{4} \mathrm{~s}$ photon ${ }^{-1}$.

On the other hand, the upconversion process in UCNPs can be described by the following model.

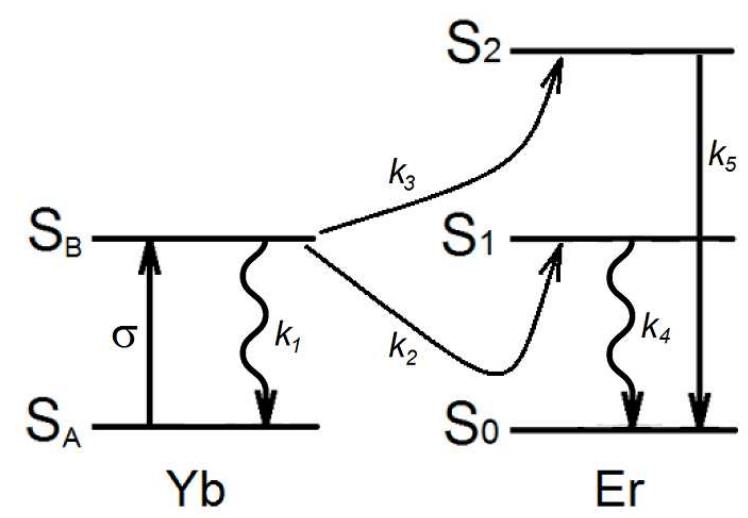

Figure S6: Scheme of the Yb-Er upconversion mechanism.

This model is rather more complex than that described in the main text, but allow us to take into account the absolute absorption parameters of the UCNPs. The absorption is given mainly through $\mathrm{Yb}^{3+}$ ions, which populate the excited state $\mathrm{S}_{\mathrm{A}}$ through a onephoton process with cross section $\sigma$. This state can perform heteronuclear ETU, promoting $\mathrm{Er}^{3+}$ ions from $\mathrm{S}_{0}$ to $\mathrm{S}_{1}$ or from $\mathrm{S}_{1}$ to $\mathrm{S}_{2}$. There is a nonradiative depopulation of $\mathrm{S}_{1}$ and an emissive depopulation from $\mathrm{S}_{2}$.

The set of equations that rules this system is given by: 


$$
\begin{aligned}
& \frac{d N_{\mathrm{A}}}{d t}=-I_{0}\left(1-\exp \left(-\sigma N_{\mathrm{A}}\right)+k_{1} N_{\mathrm{B}}+k_{2} N_{\mathrm{B}} N_{0}+k_{3} N_{\mathrm{B}} N_{1}\right. \\
& \frac{d N_{\mathrm{B}}}{d t}=-\frac{d N_{\mathrm{A}}}{d t} \\
& \frac{d N_{0}}{d t}=-k_{2} N_{\mathrm{B}} N_{0}+k_{4} N_{1}+k_{5} N_{2} \\
& \frac{d N_{1}}{d t}=k_{2} N_{\mathrm{B}} N_{0}-k_{3} N_{\mathrm{B}} N_{1}-k_{4} N_{1} \\
& \frac{d N_{2}}{d t}=-k_{3} N_{\mathrm{B}} N_{1}-k_{5} N_{2}
\end{aligned}
$$

Solving this system using the previously fitted experimental parameters for the decay paths $\mathrm{k}_{4}$ and $\mathrm{k}_{5}$, and the reported value $\mathrm{e}^{32}$ for $\sigma$ and the number of absorbent $\mathrm{Yb}^{3+}$ sites, it is possible to estimate a set of parameters that are consistent with the known $1-2 \%$ of upconversion efficiency at saturation. ${ }^{32}$ Considering a concentration of $3 \times 10^{18} \mathrm{NP} / \mathrm{cm}^{3}$ and once having a set of parameters, $\left(\mathrm{I}_{0}=2.1 \times 10^{20}\right.$ photon.cm $\mathrm{cm}^{-2}, \sigma=2.3 \times 10^{-15}, \mathrm{k}_{1}=$ $\left.8 \times 10^{8} \mathrm{~s}^{-1} \quad \mathrm{k}_{2}=\mathrm{k}_{3}=1 \times 10^{-10} \mathrm{~s}^{-1}, \mathrm{k}_{4}=2160 \mathrm{~s}^{-1}, \mathrm{k}_{5}=1660 \mathrm{~s}^{-1}\right)$, it is possible to calculate the photon flux for a non-saturation situation in which the $S_{1}$ state is populated to a molar fraction $x_{2}=10^{-4}$. In this conditions, $\left(\mathrm{I}_{\mathrm{exc}}=42 \mathrm{~W} / \mathrm{cm}^{2}\right)$, the emission flux is about about $5 \times 10^{17}$ photons/s with an upconversion efficiency of $2.37 \times 10^{-3}$.

Solving the $2 \mathrm{P}$ equations set (vide supra) with the goal of obtaining the same emission flux under the same excitation, we obtained a value of $\sigma_{2 \mathrm{P}}=7.6 \times 10^{8} \mathrm{GM} \quad(1$ Goeppert Mayer $=10^{-50} \mathrm{~cm}^{4} \mathrm{~s}$ ) that constitute an estimation of the effective absorption cross section of our UCNPs. 